\title{
DESENVOLVIMENTO DE Copaifera langsdorffii EM UMA ÁREA DE CERRADO EM REGENERAÇÃO SOB A INFLUÊNCIA DE Melinis minutiflora
}

\author{
Juliana Ribeirão de Freitas ${ }^{1}$, Maria Inês Salgueiro Lima ${ }^{2}$ \\ ${ }^{1}$ Bióloga, Mestranda em Ciências Biológicas, UFSCAR, São Carlos, São Paulo, Brasil - juzinhabio@ gmail.com \\ ${ }^{2}$ Bióloga, Dra ${ }^{\text {., }}$ Depto. de Botânica, UFSCAR, São Carlos, São Paulo, Brasil - ines@ufscar.br \\ Recebido para publicação: 06/11/2008 - Aceito para publicação: 28/07/2009
}

\begin{abstract}
Resumo
Espécies invasoras podem interferir no desenvolvimento de plantas vizinhas pela produção de substâncias químicas e sua liberação no ambiente. O objetivo deste trabalho foi testar a interferência de Melinis minutiflora P. Beauv. sobre a germinação e o crescimento de Copaifera langsdorffii Desf. Foram estabelecidos dois grupos experimentais: sementes e plantas jovens, que foram submetidas a dois tratamentos no campo, presença e ausência da gramínea. Foram demarcadas dez parcelas circulares de $4 \mathrm{~m}$ de diâmetro. Cada grupo de cinco parcelas foi submetido a um tratamento no campo, com e sem a influência da gramínea. Nas parcelas-controle o capim foi suprimido, e nas restantes foram feitos halos, mantendo-se discos de $2 \mathrm{~m}$ de diâmetro da gramínea no centro. A altura das plantas e a emergência das sementes de $C$. langsdorffii foram monitoradas a cada 20 dias, durante seis meses. As médias das emergências foram comparadas utilizando-se teste T, e o tamanho das plantas utilizando-se o teste de Mann-Whitney. A média de emergência das parcelas controle foi de $0,412( \pm 0,85)$, e das parcelas com capim foi de 0,529 ( $\pm 1,34)$. O teste de Mann Whitney apresentou $72 \%$ de semelhança entre os tratamentos. Os resultados das comparações indicaram que não houve diferença significativa entre eles.

Palavras-chave: Sementes florestais; germinação; alelopatia; gramínea; cerrado.
\end{abstract}

\begin{abstract}
Development of Copaifera langsdorffii in a savanna regeneration area under Melinis minutiflora influence. Invasive species can interfere in the development of neighboring plants through production and release of chemicals into the environment. This study aimed to test the interference of Melinis minutiflora P. Beauv. on the germination and early growth of Copaifera langsdorffii Desf. We established two experimental groups (seeds and young plants), which were submitted to two treatments in the field: presence or absence of grass. Ten circular plots with four meters of diameter were demarcated. Each group of five was subjected to one treatment on field, with and without grass influence. In the control, all the grass was abolished and in the remaining we made halos of 2 meters of diameter with grass in the center. The height of the plants and emergence seeds of de $C$. langsdorffii were monitored at every 20 days, during six months. The averages of emergency were compared using Test $\mathrm{T}$ and the size of plants used the de Mann-Whitney Test. The average of emergency of the control plots was $0.412( \pm 0.85)$ and the circles of grass was $0,529( \pm 1.34)$. The Mann Whitney test showed $72 \%$ of similarity between the treatments. The comparison between the two treatments showed no significant differences.
\end{abstract}

Keywords: Forest seeds Germination; Allelopathy; Grasses; Brazilian savanna.

\section{INTRODUÇÃO}

Cerca de $85 \%$ do grande platô que ocupa o Brasil Central era originalmente dominado pelo cerrado, representando cerca de 1,5 a 2 milhões de $\mathrm{km}^{2}$, ou seja, aproximadamente $20 \%$ da superfície do país (PIVELLO, 2005). A grande variabilidade de habitats nos diversos tipos de cerrado suporta uma enorme diversidade de espécies de plantas e animais. Estudos recentes, como o apresentado por J. A. 
Ratter e outros autores, em "Avanços no estudo da biodiversidade da flora lenhosa do bioma cerrado", em 1995, estimam o número de plantas vasculares em torno de cinco mil (PIVELLO, 2005).

Nos últimos 50 anos, com a abertura de novas fronteiras agrícolas no Brasil, o cerrado vem sofrendo crescente ocupação por atividades pecuárias, agrícolas e extração da madeira nativa, causando sérios danos ecológicos, como erosão, perda da capacidade produtiva dos solos, fragmentação de habitats naturais, extinção de espécies nativas e redução da biodiversidade. Outra consequência dessas atividades é o surgimento de populações infestantes ou espécies invasoras.

A invasão de espécies exóticas representa um grave problema para o funcionamento dos ecossistemas e ameaça a diversidade vegetal nas unidades de conservação. No Brasil, estudos sobre invasão biológica em áreas protegidas são escassos, provavelmente porque o impacto é relativamente lento e pouco evidente nas fases de estabelecimento (MARTINS, 2004).

Dentre as invasoras mais agressivas para o cerrado, encontram-se as gramíneas africanas. Quando chegaram ao cerrado, elas encontraram condições ecológicas semelhantes às de seus habitats de origem, o que facilitou sua disseminação (D'ANTONIO; VITOUSEK, 1992).

A agressividade de algumas invasoras pode estar relacionada com o elevado poder competitivo dessas espécies. Sabe-se que uma espécie pode interferir no desenvolvimento de outras por meio da produção e liberação de compostos químicos no ambiente, um fenômeno conhecido como alelopatia (MOLISH, 1937, apud RICE, 1984). De acordo com essa definição, pode-se apontar competição e alelopatia como fenômenos distintos, uma vez que o efeito alelopático depende da adição de um composto químico ao ambiente e que a competição, por sua vez, envolve a redução no ambiente de algum fator necessário a outro organismo (RICE, 1984). Alguns pesquisadores consideram a alelopatia como parte da competição (GRACE; TILMAN, 1990), enquanto outros (RICE, 1984; PUTNAN; TANG, 1986; EINHELLIG, 1995; REIGOSA et al., 1999) consideram que a influência total que uma planta pode exercer sobre a outra - incluindo competição e alelopatia - pode ser considerada como um fenômeno para o qual Muller (1969) sugeriu o termo interferência (RICE, 1984). A definição proposta por Muller (1969) será utilizada no presente trabalho.

Compostos químicos que apresentam ação alelopática são conhecidos como aleloquímicos, os quais são comumente encontrados como substâncias voláteis, substâncias lixiviadas de tecidos vivos ou mortos, em exsudatos radiculares ou ainda em substâncias liberadas durante a decomposição de matérias vegetais, que podem ou não sofrer ação e modificação por microrganismos (EINHELLIG, 1995). Esses compostos têm ação principalmente na inibição da germinação, crescimento e desenvolvimento de plantas jovens, bem como indução de anormalidade no crescimento de plântulas (EINHELLIG, 1995).

Há poucos estudos que relacionam propriedades alelopáticas de plantas invasoras em ambientes naturais (CALLAWAY; ASCHEHOUG, 2000; TANG et al., 1995).

Embora nos últimos anos a conscientização para o problema das invasões biológicas tenha ocorrido no meio técnico-científico, ainda são escassas as pesquisas que fazem o diagnóstico dos efeitos dessas invasões biológicas no cerrado. Um desses efeitos pode ser a interferência das gramíneas exóticas na germinação e crescimento de espécies arbóreas.

Dentre as diversas espécies de gramíneas exóticas presentes em áreas protegidas de cerrado no estado de São Paulo, Pivello et al. (1999a,b) destacam Melinis minutiflora P. Beauv. como uma das espécies herbáceas de maior valor de importância nos estudos de fitossociologia, apontando também uma associação negativa entre ela e a maioria das gramíneas nativas.

O capim-gordura, como é popularmente conhecido, é uma gramínea de origem africana, perene, que apresenta via metabólica do tipo $\mathrm{C} 4$, reproduz-se tanto por sementes como vegetativamente e foi introduzida em muitos países tropicais como forrageira (MARTINS, 2004).

Uma das espécies de grande porte encontrada nas formações de cerrado e de mata ciliares do estado de São Paulo é Copaifera langsdorffii Desf. Conhecida popularmente como copaíba, copaíbavermelha e pau-d'óleo, essa espécie apresenta ampla distribuição, tendo sido observada nos estados de Goiás, Mato Grosso e Minas Gerais (POLO, 1993).

Diante do exposto, é possível que gramíneas exóticas como M. minutiflora causem interferência no desenvolvimento de espécies nativas como $C$. langsdorffii.

A hipótese testada no presente trabalho é a de que a germinação e o crescimento dessa espécie arbórea são diferentes na presença e na ausência de M. minutiflora. Assim, os objetivos deste trabalho são: 1) comparar a porcentagem de emergência e de mortalidade de sementes de $C$. langsdorffii em áreas 
com e sem M. minutiflora; e 2) comparar o crescimento de plantas jovens em áreas com e sem a gramínea.

\section{MATERIAL E MÉTODOS}

\section{Área de estudo}

O estudo foi desenvolvido em área de reserva legal do campus da Universidade Federal de São Carlos (21 ${ }^{\circ} 58^{\prime} \mathrm{S}, 47^{\circ} 53^{\prime} \mathrm{W} ; 872 \mathrm{~m}$ altitude), onde a vegetação é de cerrado stricto sensu (COUTINHO, 1978). Segundo estudo feito por Lorandi (1985), o solo local é classificado como LATOSSOLO Vermelho Amarelo-álico, horizonte A moderado, textura média, muito profundo, fase relevo plano a suavemente ondulado. O clima da região pode ser classificado, segundo a sistemática de Köppen, como de transição entre Cwai e Awi, isto é, clima tropical com verão úmido e inverno seco e clima quente de inverno seco (LORANDI, 1985).

\section{Montagem das parcelas}

Foram demarcadas aleatoriamente dez parcelas circulares de $4 \mathrm{~m}$ de diâmetro em áreas cobertas por capim-gordura. Em cinco parcelas, todo o capim foi suprimido, e elas foram usadas como controle. Nas outras cinco foram feitos halos, mantendo-se discos de $2 \mathrm{~m}$ de diâmetro com capim-gordura no centro. Em cada um dos dois tratamentos - com capim e controle -, três parcelas receberam plantas jovens de copaíba com tamanhos entre 10 e $15 \mathrm{~cm}$ de parte aérea, e as outras duas receberam sementes dessa espécie.

O número de parcelas instaladas e o número de sementes e de plantas que cada unidade amostral recebeu foram os seguintes:

- duas parcelas-controle com 26 sementes de C. langsdorffii sem gramíneas no círculo interno (Figura $1 \mathrm{~A})$;

- duas parcelas com 26 sementes de C. langsdorffii e com gramíneas no círculo interno (Figura 1 B);

- três parcelas-controle com 7 plantas jovens de C. langsdorffii sem gramíneas no círculo interno (Figura 2A);

- três parcelas com 7 plantas jovens de $C$. langsdorffii com gramíneas no círculo interno (Figura 2 B).

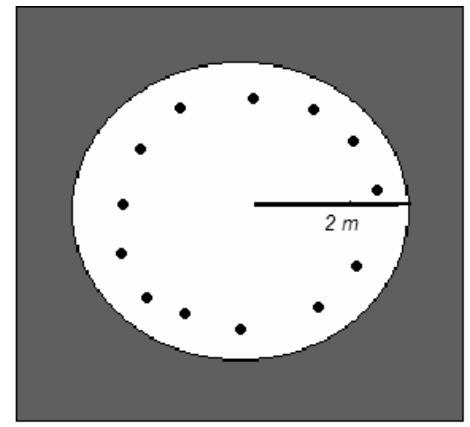

A

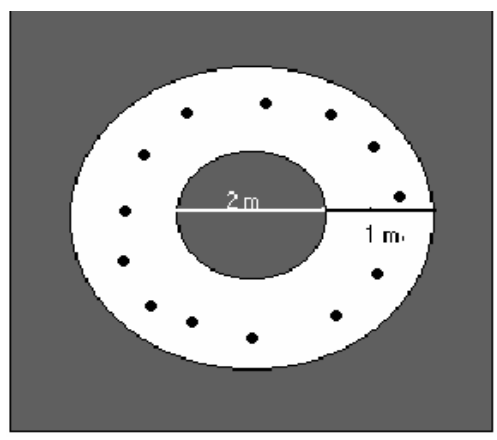

B

Figura 1. Esquema ilustrativo das parcelas com sementes de C. langsdorffii. A - parcelas-controle. B parcelas com gramíneas no centro.

Figure 1. Diagram illustrating the plots with seeds of C. Langsdorffii. A - control plots. B - plots with grasses in the center.

\section{Acompanhamento e análise dos dados}

As medidas das plantas jovens e a contagem de emergências e de plântulas mortas foram feitas a cada 20 dias, no período de dezembro de 2005 a junho de 2006. As médias das emergências nas parcelas com capim foram comparadas com seu respectivo controle por teste T (ZAR, 1999), com intervalo de 
confiança de $95 \%$. O tamanho das plantas jovens das parcelas com capim foi comparado com o tamanho das plantas das parcelas-controle pelo teste de Mann-Whitney (ZAR, 1999). As análises estatísticas foram feitas com o auxílio do programa Bioestat 3.0.

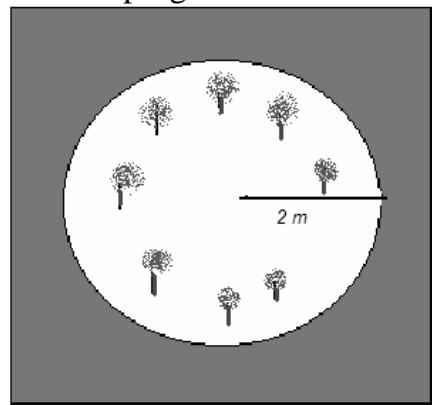

A

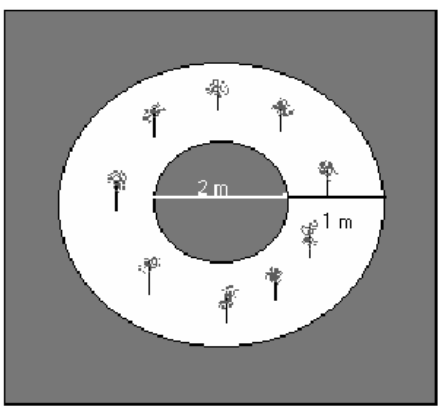

B

Figura 2. Esquema ilustrativo das parcelas com plantas jovens de C. langsdorffii. A - parcelas-controle. B - parcelas com gramíneas no centro.

Figure 2. Diagram illustrating plots with young plants of $C$. Langsdorffii. A - control plots. B - plots with grasses in the center.

\section{RESULTADOS E DISCUSSÃO}

\section{Emergência das plantas jovens}

A porcentagem de emergência observada nas parcelas-controle foi de 13,46\%. A emergência das plântulas teve início em torno do quadragésimo dia, quando foram observadas cinco plântulas, o maior número em um dia. A partir daí, o número de plântulas novas passou a diminuir (Figura $3 \mathrm{~A}$ ). Dentre as plântulas emergidas, o total de cinco morreram, sendo que quatro delas ocorreram em torno do $60^{\circ}$ dia. $\mathrm{O}$ total de plântulas mortas representa 71,43\% das emergências (Figura 4 A).

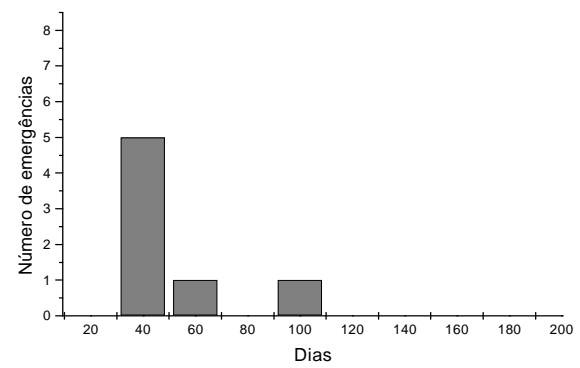

A

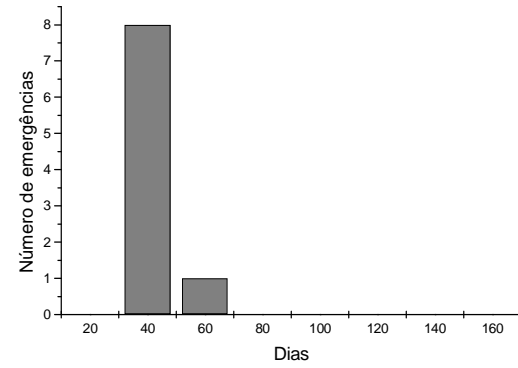

B

Figura 3. Emergência de plântulas de C. langsdorffii nas parcelas-controle de dezembro de 2005 a junho de 2006 (A) e emergência de plântulas de C. langsdorffii nas parcelas com presença de gramíneas de dezembro de 2005 a junho 2006 (B).

Figure 3. Seedlings emergence of $C$. langsdorffii in the control plots from December 2005 to June 2006 (A) and seedlings emergence of $C$. langsdorffii in the plots with grasses from December 2005 to June 2006 (B).

As primeiras emergências nas parcelas com gramíneas foram observadas no quadragésimo dia, quando foi encontrado um total de oito plântulas. A porcentagem de emergência total encontrada foi de 17,3\% (Figura 3 B). Das nove plântulas emergidas, quatro foram encontradas mortas, resultando em $44,44 \%$ de mortalidade. A duas primeiras plântulas mortas foram encontradas no $80^{\circ}$ dia, e as outras duas foram encontradas aos 100 e aos 140 dias (Figura 4 B). 
A média de emergência das parcelas-controle foi de $0,412( \pm 0,85)$, e das parcelas com capim foi de 0,529 , com desvio padrão de $\pm 1,34$. Não houve diferença significativa entre os tratamentos.

Não foi possível inferir uma relação entre taxa de mortalidade ou porcentagem de mortalidade e presença ou ausência de capim-gordura.

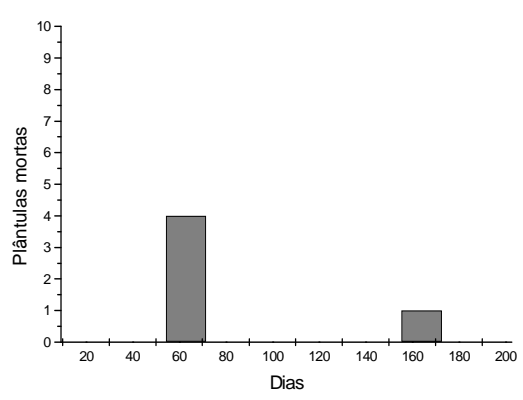

A

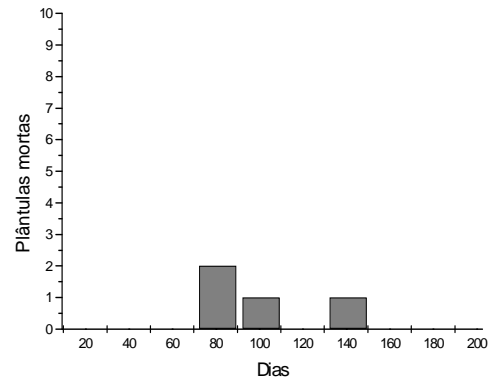

B

Figura 4. Número de plântulas de C. langsdorffii mortas nas parcelas controle de dezembro de 2005 a junho 2006 (A) e número de plântulas de $C$. langsdorffii mortas nas parcelas com presença de gramíneas de dezembro de 2005 a junho 2006 (B).

Figure 4. Number of dead seedlings of C. langsdorffii in the control plots from December 2005 to June 2006 (A) and number of dead seedlings of $C$. langsdorffii in the plots with grasses from December 2005 to June 2006 (B).

Muitas vezes, o efeito alelopático não se dá sobre a taxa de germinação (LABORIAU; VALADARES, 1976), mas sobre a velocidade de germinação ou outro parâmetro do processo. O efeito alelopático pode provocar alterações na curva de distribuição de germinação, que passa de distribuição normal para uma curtose, nas situações mais simples até distribuições erráticas, alongando a curva através do eixo do tempo (FERREIRA; AQUILA, 2000). Apesar desses parâmetros não serem facilmente observáveis em estudos de campo, apresentam consequências diretas sobre a emergência, que podem ser facilmente observadas. Os dados obtidos permitem constatar que a presença de Melinis minutiflora não causa interferência na germinação de $C$. langsdorffii em condições de campo.

Segundo Ferreira; Aquila (2000), a germinação é menos sensível aos aleloquímicos do que o crescimento das plântulas. Porém é bastante utilizada para testes de efeito alelopático, porque sua quantificação experimental é mais simples, uma vez que cada semente representa um fenômeno discreto. Segundo esses mesmos autores, foi observado que, para uma determinada quantidade de aleloquímico, o aumento da densidade de plantas diminui o efeito alelopático, embora tenha aumentado o efeito da competição. Isso acontece porque cada planta divide com as companheiras a toxicidade dos aleloquímicos, de forma a atenuar os seus efeitos.

Souza Filho et al. (2003), ao avaliarem os efeitos alelopáticos de Calopogonium mucunoides Desf. em função da densidade das sementes da planta receptora, também observaram que, com o aumento da densidade, há menor disponibilidade das substâncias para as sementes, diminuindo, consequentemente, a quantidade total de substâncias absorvidas por semente, deixando de atingir o nível requerido para promover as inibições. Além disso, esses autores verificaram que a densidade foi mais relevante para sementes grandes do que para pequenas, que requerem menor volume de água para embebição. Para eles, quanto mais pesadas eram as sementes, menores os efeitos potencialmente alelopáticos dentro de uma mesma densidade.

Gonzalez (1993), Surles et al. (1993) e Leishman; Westoby (1994) também relataram que sementes de maior tamanho têm sido correlacionadas com taxas maiores de crescimento inicial de plântulas. As sementes de maior tamanho geralmente possuem maior disponibilidade de nutrientes para o seu desenvolvimento, possuindo embriões bem formados, com maior quantidade de substâncias de reserva, sendo, consequentemente, as mais vigorosas (CARVALHO; NAKAGAWA, 2000). Copaifera langsdorffii possui sementes ovaladas, grandes (com aproximadamente $0,8 \times 0,9 \mathrm{~cm}$ ), o que as incluiria nessa categoria. 


\section{Crescimento}

O monitoramento das parcelas-controle demonstrou que houve um aumento substancial no crescimento das plantas até os quarenta dias. Após esse período, o crescimento permaneceu estável (Figura 5 A). Nas parcelas com gramíneas, foi observado um aumento crescente da altura das plantas durante aproximadamente 60 dias. Após esse período, houve um pequeno declínio, a partir do qual as plantas permaneceram aproximadamente do mesmo tamanho (Figura $5 \mathrm{~B}$ ).

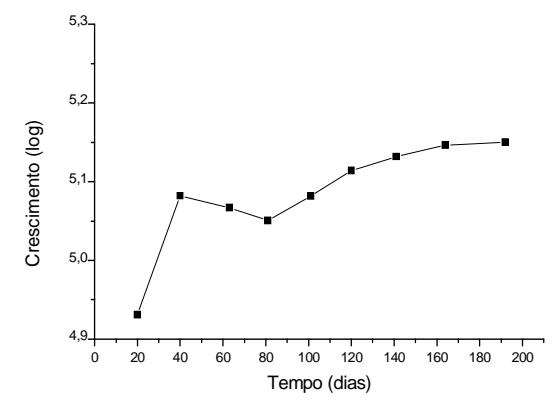

A

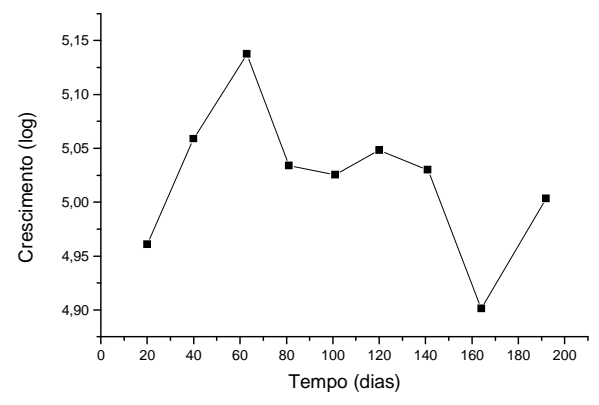

B

Figura 5. Curvas de crescimento de C. langsdorffii no período de dezembro de 2005 a junho de 2006. A - parcelas-controle. B - parcelas com presença de gramíneas no centro.

Figure 5. Growth curves of C. langsdorffii from December 2005 to June 2006. A - control plots. B plots with grasses in the center.

O teste de Mann-Whitney apresentou $72 \%$ de semelhança entre os tratamentos, demonstrando que não houve diferença significativa no tamanho das plantas.

O resultado do teste de Mann-Whitney indica que, em áreas invadidas por M. minutiflora, o tamanho de plantas jovens de $C$. langsdorffii não é modificado. No entanto, quando comparadas as curvas de crescimento dos dois tratamentos, notou-se que as plantas das parcelas sem capim-gordura apresentaram uma curva mais próxima do padrão esperado. Assim, é possível supor que haja um efeito sobre a curva de crescimento e não sobre o tamanho final.

Entretanto, podemos supor que essa gramínea invasora afete o desenvolvimento $C$. langsdorffii. O efeito alelopático pode não ser percebido na medição da altura da parte aérea, mas talvez em mudanças na biomassa seca, ou ainda na relação entre parte aérea e raiz. Estudos nesse sentido são necessários para um melhor entendimento das relações entre gramíneas invasoras e espécies nativas e, por consequência, das alterações da dinâmica de regeneração do cerrado.

\section{CONCLUSÃO}

Como os resultados estatísticos não apresentaram diferenças significativas entre os tratamentos com e sem a influência de $M$. minutiflora, pode-se concluir que a presença dessa gramínea não afeta a emergência de plântulas e nem o tamanho de plantas jovens de $C$. langsdorffii colocadas no campo com 10-15 cm de altura, durante os primeiros 200 dias após o plantio.

\section{REFERÊNCIAS}

CALLAWAY, R. M.; ASCHEHOUG, E. T. Invasive plants versus their new and old neighbors: A mechanism for exotic invasion. Science 290 (20): 521-523. 2000.

CARVALHO, N. M.; NAKAGAWA, J. Sementes: ciência, tecnologia e produção. 4. ed. Jaboticabal: FUNEP, 2000. $588 \mathrm{p}$.

COUTINHO, L. M. O conceito de cerrado. Revista Brasileira de Botânica v. 1, p. 17-23. 1978.

D'ANTONIO, C. M.; VITOUSEK, P. M. Biological invasions by exotic grasses, the grass/Fire Cycle, and global change. Annual Review of Ecology and Systematics. v. 23, p. 63-87. 1992. 
EINHELLIG, F. A. Allelopathy: current status and future goals. In: Inderjit, Dakshini, K. M. M.; Einshellig, F. A. (ed.). Allelopathy: organism, processes and applications. Washington, DC: American Chemical Society. p. 1-25. 1995.

FERREIRA, A. G.; AQUILA, M. E. A. Alelopatia: uma área emergente da ecofisiologia. Revista Brasileira de Fisiologia Vegetal. v. 12 (Edição Especial). p. 175-204. 2000.

GONZALEZ, E. J. Effect of seed size on germination and seedling vigor of Virola koschnyi Warb. Forest Management 57 (1-4): 275-281.1993.

GRACE, J. B.; TILMAN, D. (ed.). Perspectives on plant competition. Academic Press, 1990.

LABORIAU, L. G.; VALADARES, M. B. On the germination of seeds of Calotropis procera. Anais da Academia Brasileira de Ciências v. 48, p. 174-186. 1976.

LEISHMAN, M. R.; WESTOBY, M. The role of large seed size in shared conditions: experimental evidence. Funtional Ecology 8(2): 205-214. 1994.

LORANDI, R. Caracterização dos solos das áreas urbana e suburbana de São Carlos (SP) e suas aplicações. Piracicaba: ESALQ, 1985. 180 p. Tese de Doutorado (Solos e Nutrição de Plantas) - ESALQ.

MARTINS, C. R.; LEITE, L. L.; HARIDASAN, M. Capim-gordura (Melinis minutiflora P. Beauv.): uma gramínea exótica que compromete a recuperação de áreas degradadas em unidades de conservação. $\mathbf{R}$. Árvore, Viçosa, MG, v. 28, n. 5, p. 739-747. 2004.

PIVELLO V. R. Invasões biológicas no cerrado brasileiro: efeitos da introdução de espécies exóticas sobre a biodiversidade. ECOLOGIA. INFO 33. Disponível em www.ecologia.info/cerrado.htm. Acessado em 19/12/2005.

PIVELlO, V. R.; CARVALHO, V. M. C.; CARVALHO, P. F.; LOPES, A. A.; ROSSO, S. Abundance and distribution of native and alien grasses in a "cerrado" (brazilian savanna) biological reserve. Biotropica, v. 31 p. 71-82. 1999b.

PIVELLO, V. R.; SHIDA, C. N.; MEIRELLES, S. T. Alien grasses in Brazilian savannas: a threat to the biodiversity. Biodiversity and Conservation. v. 8, p. 1281-1294. 1999a.

POLO, M. Germinação, crescimento e cumarinas em Copaifera langsdorffii Desf. Campinas, UNICAMP, 1993. Tese de Doutorado.

PUTNAN, A. R.; TANG, C. S. The science of allelopathy. Wiley-Interscience publication, 1986.

REIGOSA, M. J.; ADELA, S. M.; GONZÁLES, L. Ecophysiological approach in allelopathy. Critical Reviews in Plant Sciences 18(5):577-608. 1999.

RICE, E. L. Allelopathy. Academic Press, 1984.

SOUZA FILHO, A. P. S. Efeitos alelopáticos do calopogônio em função de sua idade e da densidade de sementes da planta receptora. Planta Daninha 21(2): 211-218. 2003.

SURLES, S. E.; WHITE, T. L.; HODGE, G. R.; SURYEA, M. L. Relationships among seed weight components, seedling growth traits, and predicted field breedings values in slash pine. Canadian Journal Forest Research 23(8): 1550-1556. 1993.

ZAR, J. H. Biostatistical Analysis. Prentice-Hall, Upper Saddle River. 1999. 
FLORESTA, Curitiba, PR, v. 40, n. 2, p. 327-334, abr./jun. 2010

Freitas, J. R. de; Lima, M. I. S 